\title{
The effects of Aspergillus fumigatus challenge on exhaled and nasal NO levels
}

\author{
H.J. Stark*, J.T. Randell*, M-R. Hirvonen\#, M.K. Purokivi*, \\ M.H. Roponen ${ }^{\#}$ and H.O. Tukiainen*
}

ABSTRACT: Several studies have previously shown that exposure to indoor air microbes from moisture-damaged buildings can cause adverse health effects. Aspergillus fumigatus is one of the best-documented moulds causing health problems to those exposed.

In this study, inhalation of a commercial $A$. fumigatus solution was assessed, to establish if it would have effects on fractional exhaled ( $\mathrm{FeNO}$ ) and nasal (FnNO) nitric oxide levels and on lung function. The results were compared with placebo challenge.

A total of 28 subjects were divided into three study groups: group 1 had been exposed to occupational mould; group 2 consisted of atopic subjects; and group 3 was a control group. Some $3 \mathrm{~h}$ after $\boldsymbol{A}$. fumigatus challenge, there was a considerable increase in FeNO, and a significant difference was observed between the $A$. fumigatus and placebo inhalations. The difference was seen in all study groups. No such differences were found in the levels of FnNO or nitrite in nasal lavage fluid. Subjects reported significantly more frequent respiratory tract symptoms after the $A$. fumigatus inhalation compared with placebo challenge.

In conclusion, it was shown here that inhalation challenge of Aspergillus fumigatus elevated fractional exhaled nitric oxide levels. An increase in fractional exhaled nitric oxide may serve as an indicator of respiratory inflammation of acute mould exposure.

KEYWORDS: Acute mould challenge, Aspergillus fumigatus, exhaled nitric oxide, nasal nitric oxide

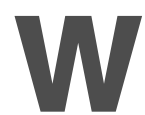
orldwide epidemiological studies have indicated that exposure to indoor air microbes present in moisture-damaged buildings is a potential cause of adverse health effects [1]. Although there is a strong association between exposure to mouldy buildings and adverse health effects in the occupants of those buildings, the aetiological factors and mechanisms behind the reported symptoms are still unclear.

An increase in fractional exhaled nitric oxide (FeNO) concentrations is associated with several inflammatory diseases, such as asthma [2]. Increased fractional nasal nitric oxide (FnNO) levels have been reported in patients with seasonal rhinitis [3]. In addition, an elevated level of nitric oxide (NO) assessed as nitrite in nasal lavage fluid (NAL) has been detected after occupational exposure to microbes from moisture-damaged buildings [4, 5]. However, no significant differences have been detected in the levels of FeNO between subjects exposed to microbes from a moisture-damaged building and control subjects [6].
It has been noted that the concentrations of airborne bacteria and fungal spores in moisturedamaged buildings are higher than in reference buildings [7]. Conversely, the cytotoxicity and inflammatory potential of the same microbes can vary between different strains [8], and are also dependent on the conditions that the microbes grow in [9]. Many of the fungi and bacteria isolated from moisture-damaged buildings produce secondary metabolites, which can be hazardous and cause health problems [10]. Among those microbes Aspergillus spp. are common toxin producers and they are also opportunistic pathogens, capable of infecting lungs if the host defence of the exposed individual is suppressed [11]. Within Aspergillus spp., Aspergillus fumigatus has been frequently isolated from the indoor air of moisture-damaged buildings where the occupants have a history of respiratory symptoms [12]. This is of particular interest, as $A$. fumigatus may cause pulmonary aspergillosis with varying degrees of severity [13]. It also produces highly toxic secondary metabolites such as gliotoxin, which can be harmful to health [14].

\section{AFFILIATIONS}

*Dept of Respiratory Medicine, Kuopio University Hospital, and \#Dept of Environmental Health, National Public Health Institute, Kuopio, Finland.

CORRESPONDENCE

H.J. Stark

Dept of Respiratory Medicine Kuopio University Hospital

PO Box 1777

FIN-70211 Kuopio

Finland

Fax: 35817172683

E-mail: harri.stark@kuh.fi

Received:

May 272005

Accepted:

July 122005 
The main goal of this study was to assess if a commercialpurified A. fumigatus extract had acute effects on FeNO, FnNO and lung function. In addition, the effects of $A$. fumigatus challenge on the production of NO, assessed as nitrite, in NAL was measured. The results of $A$. fumigatus inhalation were compared with placebo challenge (allergen diluent). Since $A$. fumigatus can cause unexpected health effects, challenging human subjects via the inhalation of microbial extracts from moisture-damaged buildings cannot be approved for ethical reasons. Therefore, an extract officially approved by the Finnish National Agency for Medicine was used in this study.

One further area of interest was whether a subject's background was associated with a different response after the challenge. Therefore, the subjects were categorised into three different groups depending on their history: a group with chronic occupational exposure to moisture-damaged building microbes (referred to later as chronic mould exposure), a group of atopic subjects and a control group.

\section{METHODS}

\section{Subjects}

A total of 28 subjects volunteered to participate in the study. All the subjects were hospital personnel and they were working in two different hospital buildings in eastern Finland. The presence of moisture and mould problems in one of the buildings was confirmed by technical and microbiological investigations. The mean age of the subjects was 45 yrs (27-60). All but one of the subjects were females. The subjects were categorised into three groups as follows. Group 1: 13 subjects working in a moisture-damaged building, who are referred to in the following text as the mould-exposed group; group 2: five atopic nonasthmatic subjects without mould exposure; and group 3: a control group including 10 nonatopic, nonasthmatic subjects with no known mould exposure.
None of the subjects had experienced upper or lower respiratory tract infections during the 6 weeks prior to the study. Those taking any medication were excluded and no hard exercise was permitted during the days when measurements were taken. Smoking was not allowed during the study. Subjects in group 1 had experienced chronic mould exposure for several years. They had normal working days during the study period. Those diagnosed bronchial asthma were excluded. The study was approved by Kuopio University Ethical Committee. The subject characteristics are shown in table 1.

\section{Protocols}

Each subject randomly underwent two challenges where they inhaled A. fumigatus or placebo solution via a nebuliser (Spira elektro 2 flowmeter ${ }^{\mathrm{TM}}$; Respiratory Care Center, Hämeenlinna, Finland) using an oronasal mask. The inhalation aerosol was generated from a 1-mL sample reservoir, with the nebulising pressure adjusted to 2 bar (flow rate $7.5 \mathrm{~mL} \cdot \mathrm{min}^{-1}$ ). The nebulisation time was $0.4 \mathrm{~s}$, causing a mean $\pm \mathrm{SD}$ volume uptake of $6.5 \pm 0.3 \mu \mathrm{L}$ of the solution per breath. The subjects inhaled each solution 60 times using controlled tidal volume respiration during a period of $\sim 5 \mathrm{~min}$, and this was followed by a rest period of $5 \mathrm{~min}$ and subsequent spirometric measurements.

Forced expiratory volume in one second (FEV1) was measured in triplicate before the challenges, and the greatest value was used as the baseline. Four series of 10-fold dilutions of commercial A. fumigatus allergen (ALK-ABELLO, Madrid, Spain) were prepared immediately before the challenges and the inhalation was started with the lowest dilution. $A$. fumigatus solution was a purified allergen product accepted to be marketed in Finland by the National Agency for Medicine. The subjects inhaled the allergen at a starting dilution of $0.01 \mathrm{mg} \cdot \mathrm{mL}^{-1}$ until the maximal concentration of

\section{TABLE 1 Subject characteristics}

Group 1 mould exposed

\section{Subjects $n$}

Age yrs

Smoking status

Current smoker

Ex-smoker

Positive skin-prick tests

Basic series or storage mites

Moulds

Serum levels IU $\mathrm{L}^{-1}$

Total $\lg E^{*}$

ECP levels

Lung function

FEV $L^{*}$

FVC L*

Diffusion capacity $\mathrm{mmol} \cdot \mathrm{min}^{-1} \cdot \mathrm{kPa}^{-1}$

\section{3}

$48(40-56)$

0

2

5

$1^{\#}$

$57.8(9.3-112.0)$
$14.5(5.4-26.3)$
$2.78(96)$
$3.37(95)$
$8.7(116)$

$247.7(52.2-757.0)$

$15.9(8.0-40.9)$

$3.56(105)$

$4.16(100)$

11.9 (138)
Group 3 controls

\section{0}

$42(27-57)$

Data are presented as mean (range) or mean (\%), unless otherwise stated. Ig: immunoglobulin; ECP: eosinophil cationic protein; FEV 1 : forced expiratory volume in one second; FVC: forced vital capacity. ${ }^{\#}$ : Geotrichium candidum; *: $p<0.05$ between groups $(1,2$ and 3$)$ using one-way ANOVA; ${ }^{*}: p<0.005$ between groups $(1,2$ and 3$)$ in one-way ANOVA. 
$10 \mathrm{mg} \cdot \mathrm{mL}^{-1}$ was administered. FEV1 was measured $5 \mathrm{~min}$ after each inhaled concentration. The difference between the baseline and lowest FEV1 was observed. The allergen diluent (physiological salt solution) was used as placebo. The placebo challenge protocol was identical to the A. fumigatus challenge, although the concentration of the solution did not change during the challenge. The challenges were performed 1 week apart at the same time of the day.

\section{Lung function tests}

Standard spirometric values (Medikro 905 Spirometer $^{\mathrm{TM}}$, Medikro, Kuopio, Finland) and pulmonary diffusion capacity (Type 2200 Sensor Medics BV ${ }^{\mathrm{TM}}$; Sensor Medics, Bilthoven, The Netherlands) were measured according to American Thoracic Society guidelines $[15,16]$. Reference values compiled by VILJANEN et al. [17] for the Finnish population were used. Baseline levels of spirometry and diffusion capacity were measured before the exposures. Lung function measurements were repeated $24 \mathrm{~h}$ after both exposures. The levels of FEV1 were monitored during the exposures, as described above. Peak expiratory flow (PEF) values were assessed before both challenges. PEF values were observed during the challenges and 3,6 and $24 \mathrm{~h}$ after them.

\section{Symptom questionnaire}

After each inhalation of the A. fumigatus or placebo solution, the subjects filled in a standardised questionnaire with respect to upper (irritation of eyes, rhinorrhea, blocked nose, sneezing) and lower (cough, phlegm, dyspnoea) airway symptoms. The lungs of the patients were auscultated by a doctor before the exposures and after each inhalation to determine possible wheezing. The patients were asked to report if they had experienced any respiratory symptoms during the subsequent $24 \mathrm{~h}$ after the exposures.

\section{Measurement of FeNO and FnNO}

FeNO was measured using a chemiluminescence analyser (Sievers Model $280 \mathrm{NOA}^{\mathrm{TM}}$; Sievers Instruments Inc., Boulder, CO, USA), according to the manufacturer's instructions using the same protocol previously described by PUROKIVI et al. [18]. When measuring FeNO, the subjects performed a slow vital capacity manoeuvre for $30 \mathrm{~s}$ against a fixed expiratory resistance. The pressure level during exhalation was optimised by following the computer screen online to reach a constant flow rate during exhalation. Exhaled air flowed through a nonbreathing valve into a Teflon tubing system connected to the analyser. Recordings were taken using the single-breath program, and they were seen on a computer screen online. The relative SD between three exhaled samples was expected to be $<10 \%$ and the detection limit for $\mathrm{NO}$ was 1 part per billion (ppb). Measurements were made in the same laboratory under constant conditions. The chemiluminescence analyser was calibrated daily by using zero air and a certified concentration of NO.

FnNO levels were measured by an application of the fixed flow exhalation technique [19]. Two soft well-fitting nose pieces were placed at the entrance to both nostrils. The pieces were attached via a " $\mathrm{Y}$ " connector to a two-way valve, and a resistor was placed in the exhalation limb, which required a pressure of $10 \mathrm{cmH}_{2} \mathrm{O}$ to produce a flow of $100 \mathrm{~mL} \cdot \mathrm{s}^{-1}$. Subjects inhaled normal room temperature air to total lung capacity via their mouths and exhaled nasally while targeting a flow signal displayed on a computer monitor. The expiration was continued until a steady $\mathrm{NO}$ plateau, lasting at least $10 \mathrm{~s}$ was reached. The contribution of oral NO was excluded. The measurement was repeated three times and the mean value was calculated. FeNO and FnNO were measured before, and 3, 6 and $24 \mathrm{~h}$ after the challenges.

\section{NAL}

NAL was performed according to the protocol described by GRAHAM and KORAN [20] with some modifications [4]. A total of $4.5 \mathrm{~mL}$ of pre-warmed Hanks' balanced salt solution $\left(37^{\circ} \mathrm{C}\right)$ was instilled through a heat-softened catheter into the nare. During the installation process, the subject held his or her chin down toward the chest and held the catheter in place by pinching the nares closed. The cartilaginous bridge of the nose was vibrated with a neonatal percussor (Neo-Cussor ${ }^{\mathrm{TM}}$; General Physiotherapy, Inc., St. Louis, MO, USA) while the fluid was refluxed three times. The same protocol was repeated on the opposite nare. The sample was centrifuged $(425 \times g$ for $10 \mathrm{~min})$ and the cells were resuspended in $2 \mathrm{~mL}$ of the supernatant. The remaining cell suspension was incubated for $24 \mathrm{~h}$ at $37^{\circ} \mathrm{C}$ and then centrifuged $(425 \times \mathrm{g}$ for $10 \mathrm{~min})$. The supernatant and cells were frozen at $-70^{\circ} \mathrm{C}$.

\section{Analysis of NO in NAL}

$\mathrm{NO}$ in the NAL supernatant was assayed using the Griess reaction, as the stable NO oxidation product nitrite [21], as described in detail previously [4].

\section{Skin prick tests}

Atopic status was determined by ALK skin prick tests (ALK laboratories, Copenhagen, Denmark). The prick tests included 14 common environmental allergens (two house dust mites (Dermatophagoides farinae and D. pteronyssinus), birch, alder, meadow fescue, timothy, meadow grass, mugwort, dandelion, horse, dog, cat, cow and natural rubber), three storage mites (Lepidoglyphus destructor, Acarus siro and Tyrophagus putrescentiae) and 13 mould (Alternaria alternata, Cladosporium herbarum, A. fumigatus, A. versicolor, Aureobasidium pullulans, Botrytis cinerea, Chaetomium globusum, Geotrichium candidum, Mucor racemosus, Penicillium brevicompactum, P. expansum, Phoma herbarum and Trichoderma viride). Histamine hydrochloride $\left(10 \mathrm{mg} \cdot \mathrm{mL}^{-1}\right)$ and ALK allergen diluent mediums were used as positive and negative controls, respectively. Atopy was defined as at least one positive reaction with a diameter $\geqslant 3 \mathrm{~mm}$.

\section{Blood samples}

From serum samples, total immunoglobulin $(\mathrm{Ig}) \mathrm{E}\left(\mathrm{IU} \cdot \mathrm{L}^{-1}\right)$ was determined by EIA (Immulite total IgE®; Diagnostic Products Corporation, Los Angeles, CA, USA), ECP (IU $\left.\cdot \mathrm{L}^{-1}\right)$ by radioimmunoassay (RIA®; Pharmacia et Upjohn, Uppsala, Sweden) and Aspergillus fumigatus $\mathrm{IgE}\left(\mathrm{IU} \cdot \mathrm{L}^{-1}\right)$ by UniCAP-FEIA (Model Unicap 100®; Pharmacia AB, Uppsala, Sweden).

\section{Statistical methods}

Differences in changes from baseline on the course of time (e.g. $\mathrm{FeNO}, \mathrm{FnNO}$, nitrate in NAL and spirometric values) between the A. fumigatus and placebo exposures were compared by 
variance analysis for repeated measures. Logarithmic transformations were used for variables not normally distributed (FeNO, nitrite in NAL). Means between the groups were compared using one-way ANOVA and mean ranks using the Kruskall-Wallis test. The percentages were compared by using the Chi-squared test. Data are presented as arithmetic means with mean values if not otherwise stated. A p-value of $<0.05$ was considered significant in all analyses.

\section{RESULTS}

\section{Symptoms}

Symptoms of both upper (blocked nose, rhinorrhea, sneezing) and lower (cough, phlegm, dyspnoea) airways were more common during the A. fumigatus challenge when compared with placebo inhalation, although the differences were not statistically significant (table 2). Instead, during the next $24 \mathrm{~h}$ after the challenges, the subjects reported significantly more frequent airway symptoms after the $A$. fumigatus challenge when compared with placebo inhalation $(\mathrm{p}=0.014)$. Typical symptoms after the $A$. fumigatus challenge were blocked nose, phlegm and sneezing. There was no difference in the upper and lower airway symptoms reported during the 24-h followup period between the study groups. Two of the subjects also complained of fatigue in addition to respiratory tract symptoms after $A$. fumigatus inhalation.

\section{FeNO and FnNO oxide levels}

The baseline levels of FeNO did not differ significantly between the A. fumigatus and placebo inhalations (20.4 and $21.7 \mathrm{ppb}$, respectively). A significant increase in FeNO levels in all subjects was observed after A. fumigatus inhalation $(\mathrm{p}=0.002)$. Peak levels of FeNO were achieved $3 \mathrm{~h}$ after $A$. fumigatus inhalation. No similar responses were detected after the placebo inhalation and there was a significant difference between the challenges $(p<0.05)$. The difference was not dependent on which group each subject belonged to (fig. 1a). When one subject who experienced an asthmatic reaction during the A. fumigatus challenge ( $25 \%$ decrease in FEV1) was excluded, there was still a tendency, but not a significant difference, in FeNO levels between the challenges $(p=0.082)$.

The baseline levels of FnNO did not differ significantly between the A. fumigatus and placebo inhalations (129.5 and $127.0 \mathrm{ppb}$, respectively). During the $24-\mathrm{h}$ follow-up after the challenges,

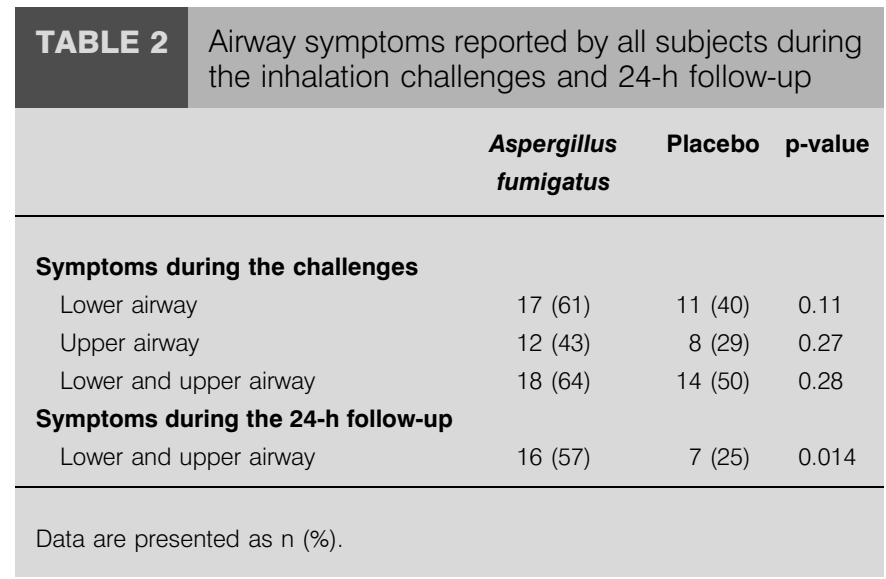
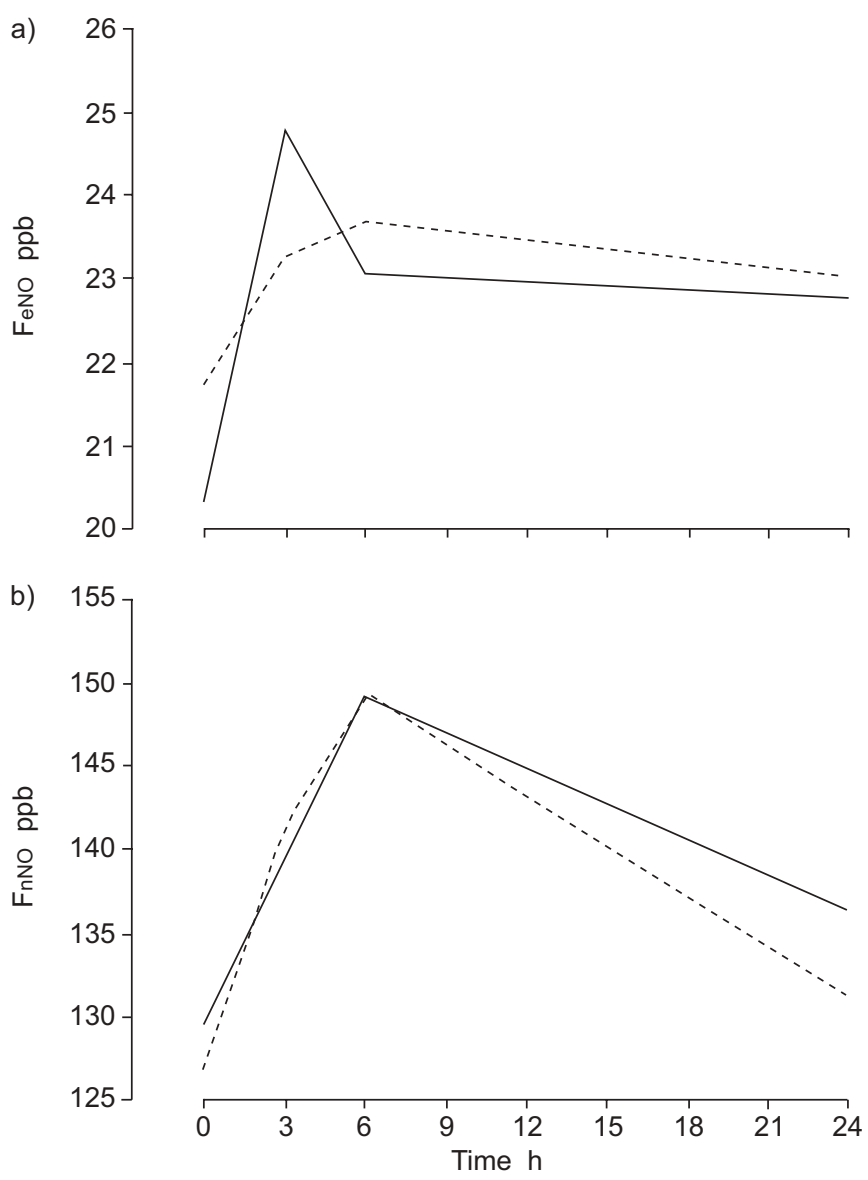

FIGURE 1. The changes in mean levels of a) exhaled (FeNO) and b) nasal ( $\mathrm{FnNO}$ ) nitric oxide (all of the subjects) after Aspergillus fumigatus ( - ) and placebo (----) challenges. There was a significant difference between the challenges in FeNO $(p<0.05)$, but not in FnNo.

the levels FnNO of all the subjects increased significantly after the placebo inhalation $(\mathrm{p}=0.003)$ and there was a tendency to changes in FnNO concentrations after the A. fumigatus challenge $(p=0.051)$. The peak levels of FnNO after both challenges were measured $6 \mathrm{~h}$ after the inhalations. The FnNO profiles after the challenges were almost identical and there was no significant difference between the challenges ( $p=0.81$; fig. $1 b$ ).

\section{Nitrite levels in NAL}

Nitrite levels in NAL did not change significantly during the follow-up time after A. fumigatus or placebo challenges. Moreover, there were no significant differences between the A. fumigatus and placebo challenges. However, there was an upward trend in nitrite levels after the A. fumigatus challenge but not after the placebo inhalation. There was a wide range of nitrite levels (table 3).

\section{Lung functions}

The baseline forced vital capacity (FVC) and FEV1\% predicted were significantly lower among the mould-exposed subjects when compared with the other groups $(p=0.046$ and $p=0.004$, respectively). The baseline pulmonary diffusion capacities were higher among the atopic individuals when compared 
TABLE 3 Nitrite concentrations in nasal lavage fluid before and after inhalation challenges

\begin{tabular}{|c|c|c|c|c|}
\hline & Before & $6 \mathrm{~h}$ & $24 \mathrm{~h}$ & p-value \\
\hline Aspergillus fumigatus & $2.34(0.28-10.23)$ & $3.33(0.22-51.29)$ & $1.88(0.60-5.13)$ & 0.519 \\
\hline Placebo & $2.78(0.37-12.02)$ & $2.65(0.68-24.55)$ & $2.10(0.60-8.71)$ & 0.673 \\
\hline
\end{tabular}

with the others $(\mathrm{p}=0.033)$. The diffusion capacities ( $\%$ pred) of the mould-exposed subjects were normal (table 1).

FEV1 decreased significantly during the A. fumigatus (3.04 to $2.941, \mathrm{p}=0.013$ ) and placebo (3.03 to $2.981, \mathrm{p}=0.029)$ challenges. There was also a significant difference in FEV1 changes between the challenges $(p=0.049)$, and the changes were not dependent on the group. However, there was one subject who exhibited a decrease of $25 \%$ in FEV1 after $A$. fumigatus challenge and a $12 \%$ decrease after placebo inhalation. When this subject was excluded, the difference in changes of FEV1 between the inhalations did not reach a statistically significant level $(p=0.320)$.

Peak expiratory flow values did not change significantly during or after the challenges, and there were no significant differences between the A. fumigatus and placebo exposures. No significant changes in spirometric values and diffusion capacity were found $24 \mathrm{~h}$ after the exposures when compared with baseline (data not shown).

\section{DISCUSSION}

In the present study, the inhalation of A. fumigatus solution evoked a significant increase in FeNO levels. The changes in FeNO levels between the $A$. fumigatus and placebo challenges also differed significantly. These findings are in line with an earlier report demonstrating that $A$. fumigatus can stimulate NO production in rat alveolar macrophages [22]. The results presented are also consistent with the findings that $\mathrm{NO}$ is increased in the NAL fluid from subjects with occupational microbial exposure, when compared with control subjects [4]. This is of interest since it is well known that FeNO concentrations increase in various inflammatory respiratory diseases, such as asthma and respiratory infections [2,23]. In a previous study, an elevation in the levels of pro-inflammatory cytokines in induced sputum was observed among occupants of a moisture-damaged building [24]. However, neither nitrite in induced sputum, nor increased FeNO levels were shown to be associated with occupational exposure to indoor air bioaerosols in another moisture-damaged building [6].

Because of the potential unexpected health effects, the extracts from moisture-damaged buildings cannot be used to challenge human subjects. The microbes isolated from moisturedamaged buildings produce secondary metabolites, such as endotoxins, which are known to mediate their inflammatory effects, but may also be cytotoxic or even mutagenic [10]. Therefore, in this study, the subjects were challenged by a commercial $A$. fumigatus solution officially approved by the Finnish National Agency for Medicine. Approval by officials was considered to be a guarantee of the safety of the inhalation extract.

PAREDI et al. [25] have previously shown a late, but not immediate, increase in FeNO concentrations among asthmatic subjects after allergen challenge. In that study, the peak levels of FeNO were achieved $10 \mathrm{~h}$ after the challenge. The first measurement point in the present study was $3 \mathrm{~h}$ after the challenges, but considering the results of PAREDI et al. [25], no earlier FeNO increase could have been anticipated. However, it is worth noting that there were no nonasthmatic subjects in the study by PAREDI et al. [25], which was not the case in the current study.

In a previous study, it was stated that $\mathrm{NO}$ alone is an insufficient biomarker of exposure to microbes in a moisturedamaged building [6]. In that and several other studies [4, 6, $24]$, the effects of chronic exposure to moisture-damaged building microbes on NO levels have been assessed. For instance, differences after vacation and occupational exposure have been compared. In the current study, NO levels were measured for 1 day after an acute exposure to only one mould often found in moisture-damaged buildings. Even though NO alone seems to be an insufficient biomarker to assess chronic mould exposure, FeNO may be appropriate for the detection of acute mould exposure.

Baseline FeNO levels were a little higher when compared with studies performed according to European Respiratory Society recommendations [26]. Conversely, the levels did not differ from previous measurements in the same laboratory [18] and the differences may be explained by the ambient NO.

The group which the subjects belonged to was not associated with the changes in FeNO. Atopic status is connected to increased FeNO levels [23], but in this study the baseline FeNO levels of the atopic subjects were not increased compared with the others. Conversely, the baseline FeNO concentrations between the groups are poorly comparable because of the small group sizes in this study. In addition, in an earlier report, those with higher baseline FeNO levels had a minor increase after allergen challenge (including $A$. fumigatus) compared to those with lower baseline values [27]. Thus, a different reaction among atopics could have been anticipated, but it was not found in this study. It is not known if chronic exposure to moulds has any effect on allergen challenge response and no association was indicated.

One of the atopic subjects had an asthmatic reaction during the A. fumigatus challenge (25\% decrease in FEV1). After exclusion 
of this subject there was not any longer a significant difference in FeNO levels between the challenges, but a distinct tendency was still to be established. The same subject had also a relatively large decrease in FEV1 after placebo challenge (12\%). This refers to bronchial hyperresponsiveness not related only to A. fumigatus; however, the decrease in FEV1 still doubled after the $A$. fumigatus challenge. This subject belonged to the atopic group. It is well-known that some nonasthmatic atopic subjects may have a subclinical asthma not previously detected.

In this study, FnNO levels increased clearly after both $A$. fumigatus and placebo challenges, and the peak levels were achieved $6 \mathrm{~h}$ after the challenges. The FnNO profiles were very similar after these two inhalations and there was no significant difference between the challenges. The changes in FnNO can be explained by normal diurnal variation [28]. It has been shown that almost all NO detected in exhaled air originates from the upper airways [29]. The intrinsic level of NO production in lower airways is much smaller and, therefore, FeNO might more easily reflect the possible changes caused by indoor air microbes. Altogether, the inflammatory changes caused by $A$. fumigatus cannot be detected by measuring FnNO.

In concordance with the detected FnNO levels, no statistically significant differences in production of nitrite in NAL were found between the A. fumigatus and placebo challenges. However, it is worth noting that the nitrite levels in NAL were increased after the $A$. fumigatus inhalation, but not after the placebo challenge, even though the difference in the changes was not statistically significant. There were also some individuals with strong responses after $A$. fumigatus challenge. The ranges in nitrite production of NAL were very large. Possibly the difference in NO of NAL between the challenges could have been detected with a larger number of subjects.

In line with increased FeNO concentrations, respiratory tract symptoms during the 24-h follow-up were significantly more common after the $A$. fumigatus inhalation when compared with placebo challenge in all the studied groups. This finding is also supported by previous studies pointing out that subjects exposed to indoor air microbes more frequently report airway symptoms than their control counterparts [5, 24].

In the current study, the FEV1 decrease during the $A$. fumigatus challenge was minor, even though there was a significant difference between the challenges in variance analysis. After exclusion of the asthmatic subject, there was no difference in FEV1 decrease between the challenges. This is in line with a previous study where no significant differences in lung functions were found between occupants with respiratory or rheumatic disorders and their controls, all working in the same moisture-damaged building [5]. Conversely, in this study, the baseline FVC and FEV1 were significantly lower among the mould-exposed subjects when compared with their controls. Furthermore, it has been noted that among waste collectors exposed to various bioaerosols, the FEV1 decreased significantly after the 4 th working day after vacation [30]. However, because of the small group sizes, the baseline FVC and FEV1 levels are not comparable in this study.

It has been suggested that exposure to indoor air fungi of moisture-damaged buildings can cause an IgE-mediated allergic reaction [31]. In the present study, none of the subjects had increased A. fumigatus-specific IgE levels, and only one subject in the group of moisture-exposed individuals exhibited a positive reaction to fungus G. candidum in skin prick tests. It has been reported that fungal allergy among those exposed in moisture-damaged schools is rare [32]. This suggests that there are also mechanisms other than allergy evoking the respiratory symptoms after exposure to indoor air fungi of moisturedamaged buildings. However, only a few fungal allergens have been accurately characterised with respect to their various morphology and antigenicity, as well as prevalent antigenic cross-reactivity [33].

There was one current smoker and four ex-smokers among the subjects in the present study. Smoking is known to decrease exhaled and nasal NO [34], but after smoking cessation oral NO rises again to normal levels [35]. The smoking subject did not smoke during the days of measurements. Thus, smoking did not have any effects on the results.

In conclusion, the current results support the assumption that inhalation of Aspergillus fumigatus solution may cause inflammatory changes in the lower airways. The rapid increase in nasal levels of nitric oxide after Aspergillus fumigatus inhalation was not dependent on whether the subjects were atopic or nonatopic or if they were exposed to indoor air microbes of a moisture-damaged building. It is possible that nasal nitric oxide measurement could potentially be applied in the assessment of acute mould exposure.

\section{ACKNOWLEDGEMENTS}

The authors would like to thank the subjects for participation in the study. R. Tukiainen is acknowledged for excellent technical assistance, E. MacDonald for revising the language and P. Halonen for help in statistical analyses.

\section{REFERENCES}

1 Bornehag CG, Blomquist G, Gyntelberg F, et al. Dampness in buildings and health. Nordic interdisciplinary review of the scientific evidence on associations between exposure to "dampness" in buildings and health effects (NORDDAMP). Indoor Air 2001; 11: 72-86.

2 Kharitonov SA, Yates D, Robbins RA, Logan-Sinclair R, Shinebourne EA, Barnes PJ. Increased nitric oxide in exhaled air of asthmatic patients. Lancet 1994; 343: 133-135.

3 Martin U, Bryden K, Devoy M, Howarth P. Increased levels of nitric oxide during nasal and oral breathing in subjects with seasonal rhinitis. J Allergy Clin Immunol 1996; 97: 768-772.

4 Hirvonen MR, Ruotsalainen M, Roponen M, et al. Nitric oxide and proinflammatory cytokines in nasal lavage fluid associated with symptoms and exposure to moldy building microbes. Am J Respir Crit Care Med 1999; 160: 1943-1946.

5 Roponen M, Kiviranta J, Seuri M, Tukiainen H, Myllykangas-Luosujärvi R, Hirvonen M-R. Inflammatory mediators in nasal lavage, induced sputum and serum of employees with rheumatic and respiratory disorders. Eur Respir J 2001; 18: 542-548.

6 Purokivi M, Hirvonen M-R, Randell J, Roponen M, Tukiainen $\mathrm{H}$. Nitric oxide alone is insufficient biomarker 
of exposure to microbes in a moisture-damaged building. Inhal Toxicol 2002; 14: 1279-1290.

7 Meklin T, Husman T, Vepsäläinen A, et al. Indoor air microbes and respiratory symptoms of children in moisture-damaged and reference schools. Indoor Air 2002; 12: $175-183$.

8 Huttunen K, Ruotsalainen M, Iivanainen E, Torkko P, Katila M, Hirvonen $M$. Inflammatory responses in RAW264.7 macrophages caused by mycobacteria isolated from moldy houses. Environ Toxicol Pharmacol 2000; 8: 237-244.

9 Hirvonen MR, Suutari M, Lignell U, Nevalainen A. Effect of growth medium on potential of Streptomyces anulatus spores to induce inflammatory responses and cytotoxicity in RAW264.7 macrophages. Inhalation Toxicol 2001; 13: 55-68.

10 Hendry KM, Cole EC. A review of mycotoxins in indoor air. J Toxicol Environ Health 1993; 38: 183-198.

11 Samson RA, Flannigan B, Flannigan ME, Verhoeff AP, Adan OCC, Hoekstra ES. Health implications of fungi in indoor environments. In: Samson RA, Flannigan B, Flannigan ME, et al., eds. Air Quality Monographs. Vol. 2. Amsterdam, Elsevier Science BV, 1994; pp. 531-538.

12 Hyvärinen A, Reiman $\mathrm{T}$, Meklin $\mathrm{T}$, Husman $\mathrm{T}$, Vahteristo M, Nevalainen A. Fungal exposure and IgGlevels of occupants in houses with and without mold problems. In: Johanning E, ed. Bioaerosols, Fungi and Mycotoxins: Health Effects, Assessment, Prevention and Control. Albany, NY, USA, Eastern New York Occupational and Environmental Health Center, 1999; pp. $166-168$.

13 Tomee JF, van der Werf TS. Pulmonary aspergillosis. Neth J Med 2001; 59: 244-258.

14 Fischer G, Muller T, Schwalbe R, Ostrowski R, Dott W. Species-specific profiles of mycotoxins produced in cultures and associated with conidia of airborne fungi derived from biowaste. Int J Hyg Environ Health 2000; 203: 105-116.

15 Standardization of spirometry, 1994 update. American Thoracic Society. Am J Respir Crit Care Med 1995; 152: 1107-1136.

16 Recommendations for DLCO. American Thoracic Society. Am J Respir Crit Care Med 1996; 154: 263.

17 Viljanen AA, Halttunen PK, Kreus KE, Viljanen BC. Spirometric studies in non-smoking healthy adults. Scand J Clin Lab Invest 1982; 159: 5-20.

18 Purokivi M, Randell J, Hirvonen MR, Tukiainen $H$. Reproducibility of measurements of exhaled NO, and cell count and cytokine concentrations in induced sputum. Eur Respir J 2000; 16: 242-246.

19 Silkoff FE, Chatkin J, Qian W, et al. Nasal nitric oxide: a comparison of measurement techniques. Am J Rhinol 1999; 13: 169-178.

20 Graham DE, Koren HS. Biomarkers of inflammation in ozone exposed humans: comparison of the nasal and bronchoalveolar lavage. Am Rev Respir Dis 1990; 142: 152-156.

21 Green LC, Wagner DA, Glogowski J, Skipper PL, Wishnok JS, Tannenbaum SR. Analysis of nitrate, nitrite and [15N] nitrate in biological fluids. Annal Biochem 1982; 126: $131-138$.

22 Gross NT, Nessa K, Camner P, Jarststrand C. Production of nitric oxide by rat alveolar macrophages stimulated by Cryptococcus neoformans or Aspergillus fumigatus. Med Mycol 1999; 37: 151-157.

23 Ricciardolo FLM. Multiple roles of nitric oxide in the airways. Thorax 2003; 58: 175-182.

24 Purokivi M, Hirvonen M-R, Randell J, et al. Changes in proinflammatory cytokines in association with exposure to moisture-damaged building microbes. Eur Respir J 2001; 18 : 951-958.

25 Paredi P, Leckie MJ, Horvath I, Allegra L, Kharitonov SA, Barnes PJ. Changes in exhaled carbon monoxide and nitric oxide levels following allergen challenge in patients with asthma. Eur Respir J 1999; 13: 48-52.

26 Kharitonov S, Alving K, Barnes PJ. Exhaled and nasal nitric oxide measurements: recommendations. Eur Respir J 1997; 10: 1683-1693.

27 Piipari R, Piirila P, Keskinen H, Tuppurainen M, Sovijarvi A, Nordman H. Exhaled nitric oxide in specific challenge tests to assess occupational asthma. Eur Respir J 2002; 20: 1532-1537.

28 Palm JP, Graf P, Lundberg JO, Alving K. Characterization of exhaled nitric oxide: introducing a new reproducible method for nasal nitric oxide measurements. Eur Respir J 2000; 16: 236-241.

29 Lundberg JO, Weitzberg E. Nasal nitric oxide in man. Thorax 1999; 54: 947-952.

30 Heldal KK, Halstensen AS, Thorn J, Eduard W, Halstensen TS. Airway inflammation in waste handlers exposed to bioaerosols assessed by induced sputum. Eur Respir J 2003; 21: 641-645.

31 Zureik M, Neukirch C, Leynaert B, Liard R, Bousquet J, Neukirch F. Sensitisation to airborne moulds and severity of asthma: cross sectional study from European Community respiratory health survey. BMJ 2002; 325: 411-414.

32 Immonen J, Laitinen $\mathrm{S}$, Taskinen $\mathrm{T}$, Nevalainen $\mathrm{A}$, Korppi M. Mould-specific immunoglobulin E antibodies in primary school students: a 3-year follow-up-study. Pediatr Allergy Immunol 2000; 14: 101-108.

33 Malling H-J. Diagnosis of mold allergy. Clin Rev Allergy 1992; 10: 213-236.

34 Persson M, Zetterström O, Agrenius V, Ihre E, Gustafsson L. Single-breath nitric oxide measurements in asthmatic patients and smokers. Lancet 1994; 343: 146-147.

35 Robbins R, Millatmal T, Lassi K, Rennard S, Daughton D. Smoking cessation is associated with an increase in exhaled nitric oxide. Chest 1997; 112: 313-318. 